\title{
Scientific updates and researches in recent years
}

\author{
Silvia Menendez-Cepero \\ Collaborator of the National Center for Scientific Research. Havana, Cuba.
}

\section{ABSTRACT}

\section{OPEN ACCESS}

\section{Citation}

Menendez-Cepero S. Scientific updates and researches in recent years [abstract]. Proceedings of the 5Th WFOT Meeting; 2016 Nov 18-20; Mumbai, India. J Ozone Ther. 2018;2(2). doi: 10.7203/jo3t. 2.2.2018.11126

\section{Academic Editor}

Jose Baeza-Noci, School of Medicine, Valencia University, SPAIN

\section{Editor}

World Federation of Ozone Therapy, Bolgna, ITALY

\section{Received}

December 9, 2017

\section{Accepted}

December 10, 2017

\section{Published}

March 4, 2018

\section{Intellectual Property}

Silvia Menendez-Cepero. This is an open access article distributed under the terms of the Creative Commons Attribution License (CC BY 4.0), which permits unrestricted use, distribution, and reproduction in any medium, provided the original author and source are credited.

\section{Author Information}

silviamenendez@infomed.sld.cu
Ozone medical applications gather strength, day by day. Ozone, in its oxidant status, administered in low doses and in a number of controlled treatments, is able to trigger the endogenous antioxidant systems achieving a redox balance.

As such, it is able to control chronic oxidative stress associated with many diseases. Moreover, levels of nitric oxide and pro-inflammatory cytokines, like tumor necrosis factor (TNF- $\mathrm{a}$ ) and interleukin 1 and 6 , were able to be modulated by ozone. Also, mitochondrial integrity and functionality was preserved, calcium homeostasis was achieved, adenosine A1 receptors were activated and activity of the superoxide dismutase enzyme was regulated [1]. In this lecture, preclinical and clinical studies of recent researches developed in Cuba are presented.

Two topics have been selected: Alcoholism [2,3] and arthritis [4,5].

In today's world, alcoholism is a serious health and social problem, affecting millions of people, without distinction of race, sex, culture or latitude. One diagnostic criterion of alcohol dependence is the appearance of a withdrawal syndrome when alcohol consumption ceases. Experimental studies have demonstrated that many consequences of withdrawal found in animals resemble those observed in humans. Such signs and symptoms of ethanol withdrawal (EW) include enhanced activity of the autonomic nervous system; body posture and motor abnormalities; hyperexcitability of the central nervous system (CNS), including sensory hyperreactivity, convulsion, anxiety, etc. Chronic ingestion of high levels of alcohol may bring about oxidative stress, associated with hepatic alterations, and of CNS, mainly due to the formation, through alcohol metabolism, of free radicals, acetaldehyde, lipid and protein oxidation and their reaction products.

The protective effects of ozone on brain injury induced by oxidative stress and behavioral changes in rats, after 2 weeks of EW, were studied [2]. Also, a clinical trial with 10 alcoholic patients (elapsed time after ethanol withdrawal: $\leq 3$ weeks) treated with ozone by rectal insufflation (ozone concentration from 20 to $35 \mathrm{mg} / \mathrm{L}$ and volumes from 100 to $150 \mathrm{ml}$ ) was performed [3]. In the preclinical study [2], 4 groups of rats ( $n=10$ each one) were settled: I-Control, II-Ethanol, III- Ethanol + Ozone $(20 \mathrm{mg} / \mathrm{L}$, dose of 1 $\mathrm{mg} / \mathrm{kg}, 10$ sessions by rectal insufflation) and IV- Ethanol + Oxygen. At the end of EW, rats were subjected to behavioral tests followed by brain tissue collection to measure markers of oxidative damage. Ozone increased food consumption, maintained water intake at the same levels as the control group and reestablished cellular redox status. Anxiety, locomotor activity and memory/learning of the rats were improved. Ozone protected the brain against oxidative injury, improving important functions of the CNS. In the clinical study [3], the results demonstrated that ozone improved $70 \%$ of the signs from Clinical Institute Withdrawal Scale (CIWA-Ar), mainly those associated to CNS. Ozone efficacy was observed in patients that required 
pharmacological treatment. Reduction of CIWA-Ar scores and the oxidative stress $(p<0.05)$ was demonstrated. In summary, ozone improved CNS functions and reduced oxidative stress in animals and patients during EW. These results suggest ozone's regulator effects on important neurotransmitters of the CNS.

With respect to arthritis, many pro-inflammatory mediators are increased in rheumatoid arthritis (RA), including Reactive Oxygen Species (ROS) such as nitric oxide NO, pro-inflammatory cytokines as tumor necrosis factor alpha (TNF-a), interleukin- 1 beta (IL-1 $\beta$ ) and other molecules.

Ozone treatment has regulatory effects on some pathological targets associated with RA [1]. Thus, the aim of this study was to investigate the efficacy of ozone therapy on joint swelling, pro-inflammatory cytokines and oxidative stress in the experimental chronic polyarthritis induced by i.p. injection of bacterial cell wall peptidoglycanpolysaccharide (PG/PS) in vivo [4]. Moreover, cytokines, nitric oxide and oxidative stress levels in spleen homogenates were evaluated.

The protocol consisted of four experimental groups $(n=20)$. Group $1(n=5)$ : Negative control, only received the needle stress into articular space, 3 times/week; Group $2(n=5)$ rats received PG/PS; Group $3(n=5)$, as group 2, but after 10 days of PG/PS administration, rats received $0.2 \mathrm{ml}$ of ozone by intraarticular injection, 3 times/week ( 3.5 weeks). Hind paws were selected by the treatments. Group $4(n=5)$, as Group 3 but ozone treatment was substituted by oxygen. In spleen homogenates ozone treatment decreased the levels of TNF- $a$ and IL- $1 \beta$ transcripts and TNF- $a$ concentration whereas NO concentration was reestablished after ozone treatment.

Besides a reduction of oxidative stress and the reestablish of fructolysine (AGE precursor) levels were achieved. All these molecules are goals of different drugs and all of them are targets of ozone therapy. Also, a clinical trial in patients with knee osteoarthritis was performed [5]. Osteoarthritis $(\mathrm{OA})$ is defined as a degenerative joint disease that causes pain, stiffness, swelling and loss of motion in the joints which is aggravated by prolonged activity. It is a process of progressive deterioration. of articular cartilage (it breaks down and becomes thin) and formation of new bone (osteophyte) at the joint surface. OA is becoming increasingly prevalent worldwide because of the combination of an aging population and growing levels of obesity. An increase level of oxidative stress occur in OA, leading to cells unable to respond effectively to normal loading regimens and potentially contributes to disease onset. The aim of this study was to evaluate the action of ozone therapy in oxidative stress parameters in synovial fluid of patients suffering of knee osteoarthritis and their clinical evolution. In 42 patients, ozone was administered rectally (ozone concentrations between 25 and $40 \mathrm{mg} / \mathrm{L}$ and volumes of 100 to $200 \mathrm{~mL}, 20$ sessions) and by intra-articular applications. Synovial fluid was extracted for the measurement of parameters associated with oxidative stress. Also, evaluation of the joint capacity, pain, and ultrasound imaging were performed. Combined ozone therapy produced an intra-articular redox balance and a significant reduction of pain, with a maintained satisfactory response in $80 \%$ of patients in 1-year follow-up. Reduction of oxidative stress by ozone not only decreased ROS concentrations, which are otherwise able to cause degeneration in joint cartilage, but also reduced metalloproteinase activities preventing their participation in a degeneration of the extracellular matrix.

In both topics studied, alcoholism and arthritis, adenosine and redox status are therapeutic targets of medical ozone. Also, an increase in the quality of life of these patients treated with ozone therapy was observed, without the presence of side effects. 
Keywords: ozone therapy, ethanol withdrawal, oxidative stress, central nervous system, arthritis, knee osteoarthritis, inflammation, synovial fluids, pain.

\section{References:}

1. Menendez S, Leon OS, Fernandez JL, Copello M, Weiser MT. Advances of Ozone Therapy in Medicine and Dentistry. La Habana, Cuba: Palacio de las Convenciones; 2016. 513 p. ISBN 978069278138859999.

2. Diaz MT, Fraga A, Dranguet J, Mallok A, Viebahn R, Menendez S, et al. Ozone oxidative postconditioning protects against the injury associated with alcohol withdrawal syndrome in rats. Ozone Sci Eng. 2012;34(6): 425-431.

3. Diaz MT, Fraga A, Dranguet J, Mallok A, Viebahn R, Menendez S, et al. Ozone therapy ameliorates nervous system disorders and oxidative stress in patients during ethanol withdrawal. A pilot study. Ozone Sci Eng. 2012;34(6):432-437.

4. Dranguet J, Fraga A, Díaz MT, Mallok A, Viebahn R, Menendez S, et al. Ozone oxidative postconditioning ameliorates joint damage and decreases pro-inflammatory cytokine levels and oxidative stress in PG/ PS-induced arthritis in rats. Eur J Pharmacol. 2013;714:318-324.

5. Calunga JL, Menendez S, Leon R, Chang S, Guanche D, Balbin A, et al. Application of Ozone Therapy in Patients with Knee Osteoarthritis. Ozone Sci Eng. 2012;34:469-475. 